\title{
LA ADOPCIÓN DE PERSPECTIVA Y LA COMUNICACIÓN DE LA RESPONSABILIDAD SOCIAL CORPORATIVA: UNA REVISIÓN CONCEPTUAL*
}

\author{
MIGUEL FERNANDO REYES VELASCO*** \\ HÉCTOR AUGUSTO RODRÍGUEZ OREJUELA****
}

Fecha de recibido: 5 de junio de 2017

Fecha de aprobado: 14 de junio de 2018

\begin{abstract}
Resumen
Este es un primer producto en el marco de una investigación en curso enfocada en las comunicaciones de la responsabilidad social corporativa (RSC). Las empresas cometen fallos en los procesos de comunicación de la RSC, uno de ellos es no conseguir la sensibilización de los consumidores con respecto a estas actividades de RSC, precisamente por la falta de comunicación de los programas o por la mínima conciencia de los consumidores de la importancia de las actividades de RSC. Encontrar la manera más efectiva de mantener informados a los consumidores, sin perder la credibilidad, desempeña un papel importante en los objetivos estratégicos de la RSC. En el marco del modelo de comunicaciones de la RSC, surge la adopción de perspectiva (empatía cognitiva, role-taking, perspective-taking) como un concepto que hasta ahora no ha sido analizado a la luz de la literatura en las comunicaciones del marketing y el comportamiento del consumidor, como alternativa en el diseño del contenido de mensajes relacionados y dirigidos a influir en los stakeholders. Se hace una revisión de los conceptos y sus alternativas.
\end{abstract}

Palabras clave: responsabilidad social corporativa (RSC); adopción de perspectiva; empatía; comunicación; marketing.

* Este artículo de revisión es resultado de la tesis doctoral La adopción de perspectiva en la comunicación de la responsabilidad social corporativa y su impacto en el comportamiento del consumidor. Grupo de Investigación en Marketing de la Universidad del Valle.

* Candidato a doctor en Administración de la Universidad del Valle, Colombia. Profesor de la Facultad de Ciencias de la Administración de la Universidad del Valle, Colombia. Correo electrónico: miguel.f.reyes@correounivalle.edu.co

**: Doctor en Ciencias de la Empresa de la Universidad de Murcia, España. Profesor titular y de tiempo completo de la Facultad de Ciencias de la Administración de la Universidad del Valle, Colombia. Correo electrónico: augusto.rodriguez@correounivalle.edu.co 


\title{
PERSPECTIVE-TAKING AND CORPORATE SOCIAL RESPONSIBILITY COMMUNICATION: A CONCEPTUAL REVIEW
}

\begin{abstract}
This is the first product of an ongoing research project focused on Corporate Social Responsibility (CSR) communication. It is stated that companies make mistakes in CSR communication processes, one of which is not to raise awareness of CSR activities among consumers because of their failure to disseminate programs or consumer's disregard of the importance of CSR activities. Finding the most effective way to keep consumers informed, without losing credibility, plays an important role in the strategic objectives of CSR. In the framework of the CSR communication model, perspective-taking (cognitive empathy, role-taking) emerges as a concept that has not yet been analyzed in light of the literature on marketing communication and consumer's behavior, as an alternative in designing contents related to and aimed at influencing stakeholders. Some concepts and their alternatives are reviewed.
\end{abstract}

Keywords: communication; corporate social responsibility (CSR); empathy; marketing; perspective-taking.

\section{A ADOÇÃO DE PERSPECTIVA E A COMUNICAÇÃO DA RESPONSABILIDADE SOCIAL CORPORATIVA: UMA REVISÃO CONCEITUAL}

\begin{abstract}
Resumo
Este é um primeiro produto de uma pesquisa em andamento focada nas comunicações da responsabilidade social corporativa (RSC). Pomering e Dolnicar (2009) expressam que as empresas cometem falhas nos processos de comunicação da RSC, uma delas é não conseguir a sensibilização dos consumidores sobre essas atividades de RSC, justamente pela falta de comunicação dos programas ou pela mínima consciência dos consumidores da importância das atividades de RSC. Encontrar a maneira mais efetiva de manter os consumidores informados sem perder a credibilidade desempenha um papel importante nos objetivos estratégicos da RSC (Bhattacharya e Sen, 2004). No âmbito do modelo de comunicações da RSC, surge a adoção de perspectiva (empatia cognitiva, rol taking, perspective taking) como um conceito que até agora não foi analisado à luz da literatura nas comunicações do marketing e do comportamento do consumidor, como alternativa no desenho do conteúdo de mensagens relacionadas e dirigidas a influenciar os stakeholders. Nesse sentido, faz-se uma revisão dos conceitos e de suas alternativas.

Palavras-chave: responsabilidade social corporativa (RSC); adoção de perspectiva; empatia; comunicação; marketing.

Reyes Velasco, M. F., y Rodríguez Orejuela, H. A. La adopción de perspectiva y la comunicación de la responsabilidad social corporativa: una revisión conceptual. Revista de la Facultad de Ciencias Económica: Investigación y Reflexión, XXVI(2), DOI: https://doi.org/10.18359/ rfce. 2888
\end{abstract}

JEL: M14, M31. 


\section{Introducción}

La responsabilidad social corporativa (RSC) constituye un reto muy importante para las organizaciones de hoy. Los modelos de estandarización y los desafíos de los mercados globales enfocan en las empresas, no solo la búsqueda de maximizar sus ganancias, sino también la de alcanzar la dotación de capacidades que les permitan mejores posiciones en los mercados. Según Battacharya y Sen (2003) y Du, Bhattacharya y Sen (2010), la RSC les permite a las empresas alcanzar características diferenciadoras en el mercado y una ventaja competitiva. Pero no basta con dar importancia a la estrategia de RSC, sino que es aún más relevante comprender la importancia que tiene para las empresas realizar una efectiva comunicación de la RSC a las partes interesadas. En este sentido, es importante identificar los medios y las herramientas que le permitan a la empresa comunicar y alcanzar altos niveles de efectividad en sus actividades de RSC dirigidas a sus consumidores, analizando los diversos aspectos de estas decisiones, desde el contenido del mensaje hasta los canales de comunicación utilizados, y así lograr beneficios para sus marcas y productos (Pomering y Dolnicar, 2009).

En la planificación de las comunicaciones de la RSC y del diseño del contenido del mensaje, es necesario analizar los motivos, el compromiso de la empresa, el impacto y la congruencia (Du, Bhattacharya y Sen, 2010). La psicología cognitiva y social aporta el concepto adopción de perspectiva (entendida como la habilidad de ponerse en el lugar del otro o la habilidad para entender la interacción entre uno mismo y otros (Flavell et al., 1968) como herramienta básica en el diseño de mensajes comunicacionales, con lo cual da respuesta anticipada a las reacciones de los consumidores, percibiendo necesidades, motivaciones $\mathrm{u}$ opiniones. En la adopción de perspectiva, se logra intercambiar información, recomendaciones, consejos, advertencias o referencias relativas a productos, servicios, situaciones y marcas, así como a cambios en los patrones de comportamiento y consumo (Devoldre, Davis, Verhofstadt y Buysse, 2010).
A continuación, haremos un recorrido y análisis de la evolución histórica de los conceptos, su aplicación, como de las alternativas que se presentan desde la literatura científica como un gap (vacío) para futuras investigaciones (Du, Bhattacharya y Sen, 2010).

\section{Metodología}

Esta reflexión científica es el resultado de una revisión de literatura en el marco de la investigación de la RSC y la implementación de la adopción de perspectiva como herramienta para la divulgación efectiva de mensajes de RSC. El cambio de conductas del consumidor busca generar efectos hacia un comportamiento prosocial que logre aunar y unificar los valores personales de los consumidores con los valores empresariales reflejados en las estrategias comunicacionales de la RSC.

En este sentido, se ha acudido a bases de datos científicas tales como Scopus, Gale, EBSCO, Emerald, Elsiever, Scielo, Sage, Scimago, Springer, entre otras, las cuales nos han proporcionado acceso a revistas y artículos del orden científico clave para la indagación del tema y su presente reflexión. Este artículo permite abordar en la literatura elementos conceptuales que no están referenciados hasta el momento, específicamente en lo concerniente a adopción de perspectiva, la RSC congruente y no congruente, el comportamiento prosocial, la satisfacción, la identificación en los sectores objeto de estudio y sus herramientas implementadas. En un periodo de tres años desde 2015 , se ha investigado en la temática, situación que ha permitido la formulación de esta propuesta investigativa en el marco de la formación doctoral, como iniciar la fase empírica y la publicación próxima de resultados cuantitativos derivados del fenómeno.

\section{La responsabilidad social corporativa}

La investigación relacionada con la RSC ha venido desarrollándose desde hace más de sesenta años, de modo que es un tema de gran interés por parte de la comunidad académica y empresarial. El concepto $R S C$ surge en la década de los cincuenta, dentro de los contextos organizacionales y empresariales 
norteamericanos. Bowen (1953) hace la primera definición formal de la RSC y la relaciona con acciones deseables emprendidas por parte de empresa, en la búsqueda de un beneficio para la sociedad. La RSC es de carácter voluntario y un medio para aliviar los problemas económicos y alcanzar de mejor forma los objetivos organizacionales. Friedman (1962) la define como los tipos de responsabilidades que tiene la empresa con la sociedad, aunque no clarifica de qué tipo. Hoy en día, esta responsabilidad social es ampliamente reconocida como una herramienta estratégica que permite a las empresas obtener ventajas competitivas en muchos ámbitos de actuación de la empresa (Caroll, 1999; Porter y Kramer, 2006).

A lo largo de los años, la definición de la RSC ha tenido singulares acepciones y campos de análisis: en la década de los sesenta, los intereses de la investigación se centran más en la ética y en las discusiones frente a la moralidad de los resultados financieros (Moura-Leite y Padgett, 2011), mientras que en la década de los setenta, como resultado de los intereses y de las demandas económicas y sociales de la época, muchos autores centraron sus estudios en cómo encontrar un punto de equilibrio para la aplicación de la RSC, con el propósito de que las demandas y la filosofía de la RSC no entraran en conflicto con los intereses económicos del negocio. Autores como Ackerman (1973) plantean su análisis con relación a la estructura interna de las organizaciones y los mecanismos de integración para gestionar los problemas sociales en la empresa. Caroll (1979) la define como expectativas que la sociedad tiene sobre las organizaciones, responsabilidades que abarcan los campos económico, jurídico, ético y discrecional; sin embargo, no esclarece a qué tipo de responsabilidades en particular hace referencia. Por otra parte, Sethi (1975) propone tres dimensiones para el análisis de la RSC: obligaciones del orden social (como respuesta a las variables ambientales y de mercado), responsabilidades sociales (referidas a normas y valores sociales) y sensibilidad social (voluntariedad social donde la empresa se adapta a las necesidades de la sociedad).

En la década de ochenta, como resultado de la confluencia de factores sociales, políticos y económicos, surgen enfoques de la RSC que proponen una mirada del concepto desde los stakeholders, identificando grupos de interés que influyen en la actividad de la empresa (consumidores, proveedores, competencia, instituciones gubernamentales, comunidad, medios de comunicación, activistas, grupos de interés en general) (Freeman, 1984; Yang y Rivers, 2009).

Para la década de los noventa, Wood (1991), con el objetivo de la medición de la RSC, determina tres categorías estructurales: a) principios estructurales de responsabilidad social, los cuales son los componentes del proceso de RSC; b) de legitimidad, en la que las empresas que abusan del poder que les otorga la sociedad lo perderán; y c) de responsabilidad pública y discreción de gestión, para la cual los empleados de la empresa son agentes morales de la RSC. Este también advierte que las empresas se encuentran divididas en cuatro grupos con relación a su forma de actuar frente a la sociedad y sus obligaciones en la RSC: a) empresas reactivas, que solo actúan cuando lo requieren; b) empresas proactivas, que se preparan y aportan desde su propia iniciativa; c) empresas defensivas; y d) empresas cómodas, que sin importar los resultados se amoldan de acuerdo con los intereses sociales.

Además, Watts y Holme (1999) describen la RSC como actividades sostenibles que benefician el empleo, las familias, el desarrollo económico, la sociedad y la comunidad en la búsqueda de una mejor calidad de vida.

A partir del inicio del presente siglo, la RSC se considera como una importante cuestión estratégica asociada a la sostenibilidad ambiental y social, como fuente de legitimidad institucional y legal, analizando el impacto positivo de las estrategias de comunicación en los consumidores y sus patrones de comportamiento (Du, Bhattacharya y Sen, 2010).

La Commission of the European Communities (2001) unifica las definiciones en un concepto, en el que describe la RSC como la integración social ambiental de sus negocios y operaciones y la integración voluntaria de sus stakeholders. Por otra parte, la International Organization for Standardiza- 
tion (ISO, 2010), que generó la ISO 26000:2010, define la RSC como la "responsabilidad de una organización ante los impactos que sus decisiones y actividades ocasionan en la sociedad y el medio ambiente, mediante un comportamiento ético y transparente (la traducción es mía)" (p. 3). Sus valores esenciales están soportados por gobernanza de la organización, derechos humanos, prácticas laborales, medio ambiente, prácticas justas de operación, asuntos de consumidores, participación y desarrollo de la comunidad.

La tendencia en el ámbito investigativo de la RSC advierte de la singularidad de los estudios del consumidor y su comportamiento frente a las comunicaciones de la RSC. Los resultados como práctica estratégica organizacional son reconocidos a partir de los efectos positivos que generan en el comportamiento del consumidor (Brown y Dacin, 1997; Creyer, 1997; Marín y Ruiz 2008; Sen y Bhattacharya, 2001; Sen, Bhattacharya y Korschun, 2006). También Brown y Dacin (1997) y Becker-Olsen (2006) indagan las variables del comportamiento del consumidor y cómo estas influyen en la eficacia de las acciones de la RSC. Los resultados se ven reflejados en la lealtad del consumidor, intención de compra e identificación. En este sentido, Sen y Bhattacharya (2001) estudian cómo las consecuencias de las actividades de la RSC están relacionadas con el punto de vista y las creencias de los consumidores respecto de la motivación de la empresa para llevar a cabo dichas actividades. Los resultados indican que los consumidores evalúan positivamente las empresas cuando perciben un fit (congruencia) entre los objetivos de la empresa y las actividades de la RSC.

Klein y Dawar (2004) analizan el papel que desempeña la reputación de la empresa en las comunicaciones de la RSC en contextos de crisis de un producto. Los consumidores están más propensos a atribuir la culpa a la empresa en una crisis cuando esta tiene un pobre historial en materia de RSC. Cuando ese historial es positivo, sin embargo, los consumidores adjudican la culpa a otras razones y la imagen de marca se mantiene intacta. La RSC actúa como una protección frente a momentos de crisis de las empresas. También, Klein y Dawar mencionan que las actividades de RSC pueden ser asociadas a las capacidades corporativas, podrían tener un impacto y actitud positiva en la evaluación de la imagen de la empresa y generarían un comportamiento de mayor lealtad hacia el producto debido a una mejor imagen de la marca y de los productos de la empresa.

Por su parte, Dahlsrud (2006) propone cinco dimensiones o campos de acción para el análisis de la RSC: a) la dimensión ambiental o de ambiente natural (limpieza del medioambiente, operación de negocios ambientales), b) la dimensión social (contribución para una mejor sociedad, integración social de operación de negocios y su impacto en la comunidad), c) la dimensión económica descrita desde el punto de vista de los negocios (contribución económica al desarrollo), d) la dimensión stakeholders o grupos de interés y e) la dimensión voluntariedad de acciones no prescritas por la ley (interacción de las organizaciones con sus empleados, clientes y comunidad).

Asimismo, autores como Ellen, Webb y Mohr (2006) se cuentan como unos de los más destacados en el análisis de la percepción de los consumidores frente a la RSC, a la vez que analizan las motivaciones que llevan a una empresa a comprometerse con actividades de RSC. Estos autores afirman que los consumidores perciben que las acciones que motivan a la empresa son acciones egoístas de carácter estratégico, acciones de ayuda y apoyo en beneficio de otros y acciones promovidas por los grupos de interés. Las acciones egoístas y las que son promovidas por los grupos de interés son percibidas con sentimiento negativo por los consumidores, a diferencia de las acciones estratégicas de ayuda o beneficio que generan sentimientos positivos en los consumidores. Otro hallazgo se centra en que los consumidores aprenden cada vez más sobre la RSC y las motivaciones de las empresas, por lo que cada vez están más dispuestos a adoptar una perspectiva de ganar-ganar, basados en la creencia de que las iniciativas de RSC pueden y deben servir tanto a las necesidades de la sociedad como a la empresa.

Yoon, Gürhan-Canli y Schwarz (2006) cuestionan el fenómeno en razón de la percepción de la sinceridad y los motivos que llevan a la empresa a hacer activi- 
dades de RSC y de cómo estos influyen en la eficacia de las actividades de RSC, analizando dicha eficacia desde el punto de vista del posicionamiento de la marca y de la satisfacción del consumidor. Estas actividades mejoran la imagen de una empresa cuando los consumidores atribuyen motivos sinceros y son ineficaces cuando no se percibe sinceridad por parte de la empresa. Los resultados de este trabajo muestran que los consumidores utilizan la información de la RSC para inferir sobre la calidad del producto solo cuando las actividades de RSC de la empresa son relevantes y son evidentes en los resultados financieros del negocio. Por su parte, Walker et al. (2010) coinciden en el análisis e indagan las razones que llevan a los consumidores a construir sus percepciones de los programas de RSC, pero esta vez bajo la lupa de la teoría de las atribuciones, que explica la manera en que las personas asignan una razón de causalidad, es decir, la culpa o el mérito frente a la comisión de los acontecimientos, y tiene como referente inicial el comportamiento del individuo y el de otros (Jones y Kelly, 1967). Aquí, se infiere que existen ciertas razones o asociaciones del consumidor que lo llevan a percibir las actividades de comunicación de la RSC de la empresa y a brindar cierta favorabilidad a estos programas (Du, Bhattacharya y Sen, 2007; Ellen, Webb y Mohr, 2006; Sen, Bhattacharya y Korschun, 2006; Webb y Mohr, 1998).

Du, Bhattacharya y Sen (2010), según su modelo de maximización y retornos en las comunicaciones de la RSC, expresan la importancia de la comunicación en el comportamiento del consumidor, en especial del contenido y del diseño de los mensajes, los canales de difusión, los factores de contingencia, las características de la compañía y los resultados internos (atribuciones, identificación y confianza) y externos (lealtad, productividad e inversiones de capital) (Barasch y Berger, 2014).

A su vez, Ruiz de Maya et al. (2015) encuentran que los consumidores obtienen una mejor percepción, actitud hacia la empresa e identificación con dicha empresa, a partir de las actividades y comunicaciones de la RSC, cuando se promueve e involucra la participación del consumidor en las campañas de RSC. Es muy importante que la empresa dé a conocer a sus mercados sus objetivos y sus actividades de RSC (Andreu, Casado-Díaz y Mattila, 2015; Marín y Ruiz, 2008; Sen y Bhattacharya, 2001; Sen, Bhattacharya y Korschun, 2006).

Sheehy (2015) expone un enfoque estratégico de la RSC en la que se evidencian como prioritarios los beneficios económicos de la empresa (Lantos, 2002). Por otra parte, Caroll y Shabana (2010) expresan un enfoque altruista de la RSC, el cual considera la responsabilidad de las actividades empresariales de la organización en beneficio de la comunidad. Este enfoque altruista también estima acciones del orden prosocial en actividades del voluntariado, ambientales y de rendimiento social (Harjoto y Jo, 2011; Litchfield y Gentry, 2010). Marín, Cuestas y Román (2016) también hacen alusión a las actividades de RSC que se encuentran ligadas a las ayudas comunitarias, de beneficio social y enfoque filantrópico como a las actividades que tienen objetivos estratégicos y buscan la maximización de beneficios (Du, Bhattacharya y Sen, 2010).

Diversos autores enfocan sus esfuerzos en el análisis en la relación entre las variables del marketing y la RSC (Bhattacharia y Sen, 2003; Brown y Dacin, 1997; Marín y Ruiz, 2008; Sen, Bhattacharya y Korschun, 2006). Los estudios en los últimos años se encuentran centrados en el consumidor, principalmente en la respuesta del consumidor ante las iniciativas de RSC (Bigné, Chumpitaz, Andreu y Swaen,2005; Marín y Ruiz, 2007).

\section{Comunicación de la RSC en la empresa}

La información y comunicación relacionada con la empresa y sus capacidades (superioridad del producto, nuevas innovaciones, atributos y características, campañas de apoyo e impulso, actividades de $\mathrm{RSC}$ ) revelan aspectos de la identidad empresarial, que no solo son fundamentales y perdurables, sino también distintivos de la organización, en virtud de su identidad empresarial (patrocinio a causas sociales, políticas de empleo, iniciativas ambientales) (Fein y Hilton, 1994).

Du, Bhattacharya y Sen (2010) expresan que la comunicación de RSC es muy importante para construir una imagen positiva de la empresa y en 
beneficio para los consumidores. A partir de la comunicación de la RSC, las empresas pueden fomentar la lealtad, convertir a los consumidores en embajadores de la empresa, lograr la identificación con la empresa y la defensa de esta. Otra consecuencia de esta actividad se relaciona con un incremento en la disposición a pagar un mejor precio por el producto, un boca a boca positivo y una participación de los consumidores con la empresa en el desarrollo de producto y sus comunicaciones (Du, Bhattacharya y Sen, 2007).

Para Pomering y Dolnicar (2009), las organizaciones obvian el desarrollo de estrategias comunicacionales que propendan a la sensibilización de los grupos de interés frente a las actividades de RSC (Bhattacharya y Sen, 2004). En este sentido, Du, Bhattacharya y Sen (2010) reafirman la importancia de la comunicación de la RSC y proponen que el mensaje debe incluir aspectos como el compromiso de la empresa con una causa, el impacto que tendrá dicha causa, el porqué la empresa se involucra en una determinada iniciativa (es decir, los motivos de
RSC) y la congruencia entre la causa y el negocio de la empresa. De esta forma, se logrará obtener de los consumidores reacciones positivas que influyan en las variables del marketing, como el valor para la marca, la credibilidad, la lealtad, la reputación y el reconocimiento. Además, el impacto de la RSC en las organizaciones está definido por los canales de comunicación entre la empresa y sus grupos de interés, información que debe ser difundida a través de canales adecuados que permitan dar lectura a los significados cifrados en los mensajes y actividades de la empresa (Mohr y Webb, 2005; Sen, Bhattacharya y Korschun, 2006).

En la figura 1, Du, Bhattacharya y Sen (2010) proponen un marco comunicacional para la efectiva comunicación de la RSC, en el que incluye las características por tener en cuenta en los contenidos de los mensajes, los canales de comunicación, los factores contingenciales moderadores relacionados con las características de los stakeholders, las fortalezas de la compañía o la industria y los resultados internos dirigidos a los colaboradores de la empresa.

Figura 1. Modelo de la comunicación de la RSC.

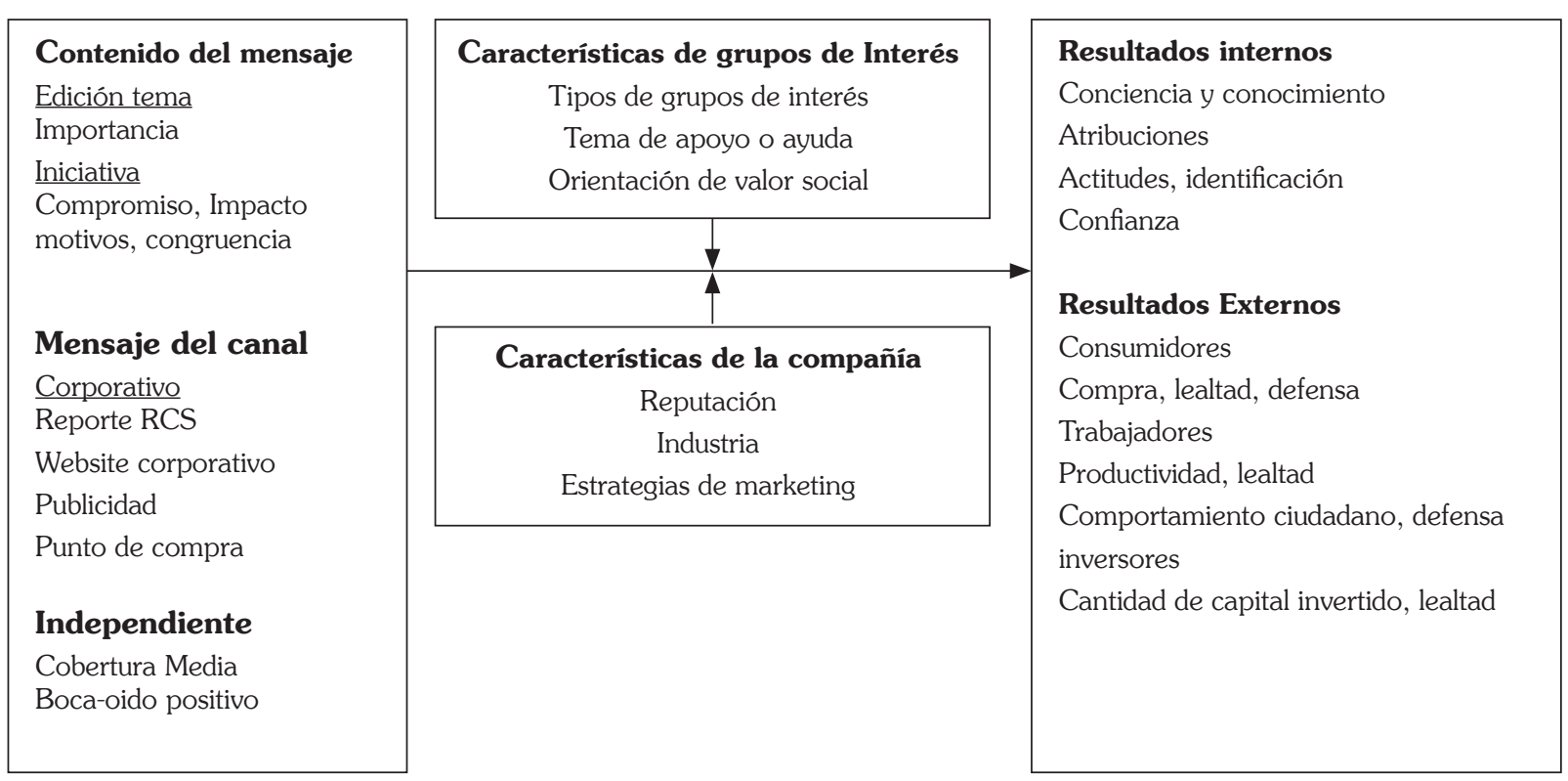

Fuente: Adaptado de Du, Bhattacharya y Sen (2010). 
El desafío clave en el diseño eficaz en la estrategia de comunicación de la RSC es la reducción del escepticismo frente a los grupos de interés, con lo cual aumentará la credibilidad a partir del compromiso en la RSC y se transmitirán favorablemente las actividades de RSC de la empresa mediante el diseño de mensajes asertivos propuestos como alternativa desde la adopción de perspectiva.

Las organizaciones emiten su comunicación a través de medios convencionales, tales como vallas, informes de gerencia, comunicados de prensa, etc. La importancia de las comunicaciones de la RSC se ve evidenciada en el número de empresas que a nivel mundial presentan sus resultados e informes de la RSC (Du, Bhattacharya y Sen, 2010). Los consumidores son un grupo importante influenciador y de poder dentro del canal de comunicación, y el boca a boca (WOM, eWOM) se configura como de gran alcance en plataformas interactivas, tales como Facebook, Instagram y Twitter. Las empresas también controlan su comunicación a través de sus medios internos, por ejemplo, su página web, club de consumidores, sindicatos, miembros del canal de distribución, con lo cual logran credibilidad de modo que son paliativos frente a críticas que podrían surgir por parte de los grupos de interés. La comunicación de la RSC es un asunto que debe ser analizado $y$ estudiado con mucha seriedad. Los resultados que se deriven de las acciones de la RSC por parte de las organizaciones pueden estar enmarcadas dentro de la sospecha, la suspicacia y la desconfianza por parte los grupos de interés, de modo que es contraproducente el efecto y, por consiguiente, el impacto en los resultados.

Basados en el modelo de comunicación de la RSC, surge la necesidad de ahondar en los mecanismos definidos desde el diseño del mensaje, su contenido y tema que puedan brindar mayor eficacia a la comunicación. Variables del orden cognitivo en el ámbito de la argumentación y afectivo, tales como la empatía, el perspective-taking o adopción de perspectiva aplicados a la argumentación serán claves como refuerzo en busca de respuestas de los consumidores y que contribuyan a la efectividad de los mensajes de RSC.

\section{Empatía, role-taking, perspective- taking, adopción de perspectiva}

La empatía hace referencia a la cualidad de "sentirse dentro". Diversos autores a lo largo de los tiempos han definido este constructo. Así, Adam Smith, en su teoría sobre los sentimientos morales de 1757, expresa que la empatía significa "ponerse en su lugar con ayuda de nuestra imaginación" (Wispé, 1986). Kohler, citado por Davis (1996), pionero del estudio cognitivo de la empatía, la define como la "comprensión de los sentimientos de los otros (la traducción es nuestra)". Mead (1934) añade a la definición el término perspectiva, de forma que adoptar la perspectiva es comprender los sentimientos del otro.

En 1947, Fenichel (Davis, 1996) habla de la empatía como la identificación con el otro, noción que será retomada más tarde con algunos planteamientos más situacionales (Igartua y Páez, 1998). Dymond (1949) desarrolla un hito importante para la definición del concepto empatía al introducir la adopción de perspectiva o role-taking como la comprensión de lo que pasa por la mente de los demás, es decir, alcanzar a identificar los estados mentales ajenos (Hogan, 1969), coloquialmente conocido como "ponerse en los zapatos del otro". Para muchos estudiosos, la empatía es la sensación de lo que otros sienten y, por tanto, empatía se superpone al concepto de contagio emocional ligado en gran medida a la teoría de la mente y la cognición social. La empatía ha sido conceptualizada a partir de varios constructos, que le han permitido ser bautizada como role-taking o la toma de rol, la toma de perspectiva $y$, finalmente, en los últimos tiempos, como adopción de perspectiva.

Para Barrett-Lennard (1981), la empatía es el concepto primario fundante, es la dimensión cognitiva a veces llamada perspective-taking o adopción de perspectiva o función cognitiva, consistente en una comprensión intelectual de la situación de otra persona. Para Davis (1980), los procesos cognitivos son denominados adopción de perspectiva (capacidad intelectual o imaginativa de ponerse en el lugar de otra persona) y los procesos emotivos son de- 
nominados comprensión emocional (capacidad de reconocer y comprender los estados emocionales de los otros). La literatura ha mostrado que las habilidades de la imaginación son una parte crítica de la asunción de roles, ya que aumentan la capacidad de considerar con flexibilidad nuevos puntos de vista (Hoffman, 1982). Además, cuando una persona es capaz de tomar el papel o la perspectiva del otro, es capaz de recuperar la información y las señales de su memoria en relación con la situación de la otra persona (Coutu, 1951; Feffer, 1959; Flavell et al. 1968; Kelley y Thibaut, 1978).

Para Davis (1980), la empatía está enfocada como una respuesta emocional que permite a una persona conectar afectivamente con otra. La empatía es a menudo considerada como una emoción de preocupación al presenciar el sufrimiento de otra persona (Batson et al., 1987). La adopción de perspectiva es la capacidad cognitiva de la empatía que hace posible considerar el mundo desde otros puntos de vista, que "permite a un individuo anticipar el comportamiento y las reacciones de los demás (la traducción es mía)" (p. 115).

Para Hoffman (1982), son seis modos mediante los cuales se lleva a cabo la empatía. Entre los seis modos, la adopción de perspectiva es el modo más avanzado. Hoffman (2000), cuya teoría del desarrollo moral ha proporcionado la visión más completa de la empatía, incluye cinco mecanismos que explican cómo un observador se inquieta al observar la angustia de un objetivo. Los cinco mecanismos son estos: a) mímica, b) condicionamiento clásico, c) asociación directa, d) asociación mediada y e) la asunción de papel.

Con una visión integradora, Davis (1996) propone que la empatía está compuesta tanto por procesos cognitivos como afectivos. Fishman y Josselson (2001) muestran cómo los procesos cognitivos son la antesala de los procesos afectivos, y que solo es posible hablar de adopción de la perspectiva si se presentan los procesos cognitivos, con su contraparte afectiva o sin ella. De esta manera, se pueden encontrar tres tipos de procesos de adopción de perspectiva distintos: a) empatía cognitiva, que incluye tanto la adopción de perspectivas como la comprensión emocional; b) contagio emocional, en el que se produce únicamente resonancia emocional; y c) empatía cognitivo-afectiva, en la que se presentan todos los procesos conjuntamente (Davis, 1996).

Existe un amplio consenso de que el dominio de la empatía contiene ambas dimensiones cognitivas y emocionales, y que la empatía emocional es la respuesta emocional a un ser $\mathrm{u}$ objeto en apuros y la empatía cognitiva es la excitación de sentir los pensamientos de los demás, también llamada adopción de perspectiva en la asunción de los puntos de vista, situaciones y roles de los otros (Davis, 1994; Garaigordobil, 2009; Thompson y Gullone, 2003). En muchos casos, la empatía ha estado estrechamente vinculada a comportamientos de ayuda y comportamiento interpersonal (Batson, 1987; Eisenberg y Fabes, 1990; Hoffman, 1982; Oswald, 1996, 2002; Penner, Dovidio, Piliavin y Schroeder, 2005).

Autores como Epley, Caruso y Bazerman (2006), Wondra y Ellsworth (2015) expresan que la toma de perspectiva es una parte de la empatía y debe considerarse empática cuando conduce al comportamiento altruista. También se asocian la toma de perspectiva, la autorregulación y la percepción de la mente en un proceso netamente cognitivo (Decety 2011; Zaki, 2014). La empatía cognitiva requiere la comprensión de los estados internos, las razones esenciales de la mente en el individuo y la validación de la emoción de los otros (Cox, Uddin, Di Martino, Castellanos, Milham y Kelly, 201; Nummenmaa et al. 2008; Saxe, 2006; Schnell et al., 2011; Shamay-Tsoory, Aharon-Peretz y Perry, 2009; Wondra y Ellsworth, 2015).

Los estudios interesados en la visión cognitiva de la empatía han empleado diferentes instrucciones que diferencian las que se referían a imaginarse a sí mismo en una situación e imaginarse al otro en otra. Por ejemplo, Davis (1996) encontró que los sujetos que tendían a puntuar más alto en adopción de perspectiva lo hacían más alto en la condición imaginarse al otro. Asimismo, existen datos que indican que el componente cognitivo de la empatía, esto es, la adopción de perspectiva, se relaciona inversamente con el nivel de agresividad 
(Richardson y Malloy, 1994). Por esto, la adopción de la perspectiva-empatía cognitiva podría ayudar a los terapeutas a comprender los pensamientos de los clientes en comunicación y en procesos educativos en la adopción de roles, y en temas educacionales los maestros podrían reconocer una falta de comprensión de los alumnos (Rogers, 1975). El debate se refiere a si la empatía cognitiva y la toma o adopción de perspectiva (es decir, teniendo la perspectiva del objetivo, la adopción de su punto de vista) son la misma construcción. Varios autores sugieren que son el mismo constructo (Coplan, 2011; Eisenberg, Fabes y Spinrad, 2006; Eklund, Andersson-Stråberg y Hansen, 2009; Fan, Duncan, De Greck y Northoff, 2011; Nakao y Itakura, 2009; Shamay et al., 2009).

Los sentimientos y las emociones se soportan sobre una amplia variedad de cosas. Algunas veces las personas sienten emociones por algo que sucede, en otras ocasiones, sienten emociones porque algo le sucede a otra persona; estas son las emociones vicarias. En la empatía y la adopción de perspectiva, sienten la misma emoción que la otra persona (Decety y Cowell, 2014a; Decety y Cowell, 2014b). Para Zaki (2014), el concepto está ligado a dos valores: la comprensión de los demás y la validación de las emociones de los demás.

Por su parte, Peng, Lee y Heeter (2010) expresan que la adopción de perspectiva por parte de un individuo va a influir en:

- Procesos comunicativos del diseño del mensaje para que sea entendido por el oyente, lo que facilita el acercamiento y el diálogo constructivo.

- Entornos cooperativos (resolución de problemas, negociación [Brion, Meisel y Selman, 1984]). Negociación interpersonal (negociaciones impulsivas y egocéntricas, de mando en un solo sentido, recíprocas de intercambio, mutuas de colaboración).

- Proceso de formación y cambio de actitudes.

- Conciencia política.

- Educación de los sujetos.
La adopción de perspectiva es aplicada en las organizaciones, en el diseño y en la argumentación para los procesos de ventas, en la formación profesional de vendedores, de personal en diversas áreas del conocimiento, en el cambio de actitudes y de comportamientos organizacionales, en las áreas de servicio al cliente y contact centers, entre otros.

Dentro del campo organizacional, surgen investigaciones que dan cuenta de la aplicabilidad del concepto. Litchfield y Gentry (2010) proponen la adopción de perspectiva como una capacidad de la organización para facilitar la integración del conocimiento, que es considerada como crucial para el aprendizaje y la innovación. Sobre la base de la investigación psicológica a nivel individual, la adopción de perspectiva podría escalar a una capacidad organizativa que puede variar en fuerza y carácter. McBane (1995), por su parte, analiza el papel que cumple la adopción de perspectiva en el contexto de las ventas, tras lo cual encontró que la adopción de perspectiva influye en el rendimiento e intercambio en las negociaciones de los vendedores. Galinsky, Magee, Gruenfeld, Whitson y Liljenquist (2008) se centran en las dinámicas de negociación, analizando las implicaciones y relaciones entre la adopción de perspectiva y la empatía (emocional). Los resultados muestran que el aumento de la adopción de perspectiva en los individuos brinda capacidades para descubrir y lograr acuerdos que permiten mejores resultados en las negociaciones. Por el contrario, la empatía no resultó tan ventajosa, y a veces era perjudicial, para llegar a un posible acuerdo y el logro de la ganancia individual.

Wieseke, Geigenmüller y Kraus (2012), en un contexto de análisis de la prestación del servicio, han definido el papel que desempeñan los empleados y clientes en la adopción de perspectiva como un constructo multidimensional que influye en la satisfacción y en la lealtad del cliente. Sus resultados muestran que la adopción de perspectiva del cliente refuerza el efecto positivo de la adopción de perspectiva de los empleados sobre la satisfacción del cliente, lo que lleva a más interacciones simbióticas. Los resultados también indicaron que los clientes empáticos son más propensos a responder a un insatisfactorio encuentro con "perdón", en el sen- 
tido de que la adopción de perspectiva es capaz de mitigar los efectos negativos de la insatisfacción del cliente propiciando lealtad. Otros autores como Delpetriche (2013) han encontrado que la adopción de perspectiva tiene un efecto positivo en el rendimiento y el éxito del vendedor. Algunos investigadores han encontrado que la empatía cognitiva no tiene ningún efecto sobre los resultados de ventas. Pero estos resultados contradictorios se han atribuido a problemas metodológicos, como la mala conceptualización y medición de la adopción de perspectiva (McBane, 1995; Pryor, Malshe y Paradise, 2013; Wieseke, Geigenmüller y Kraus, 2012).

La adopción de perspectiva ha sido conceptualizada desde el marketing y las ventas en diversos trabajos como empatía cognitiva con un mínimo componente emotivo. Autores como Aggarwal et al. (2005), Dawson et al. (1999), Lamont y Ludnstrom (1997), entre otros, son algunos de ellos. En la tabla 1, se resalta en orden cronológico la definición de empatía desde una visión cognitiva acompañada de una visión emotiva, referida como el entendimiento intelectual del estado interno de otra persona (Hogan, 1969), que se anticipa en las reacciones de la otra y que percibe sus necesidades, motivaciones u opiniones en interacciones de ventas y prestación del servicio (Devoldre, Davis, Verhofstadt y Buysse, 2010).

La adopción de perspectiva como herramienta cognitiva también ha sido perfeccionada e implementada en procesos de investigación desarrollados en otras áreas de conocimiento, como la medicina, la pedagogía (por ejemplo, en el comportamiento prosocial de niños, en procesos de formación y educación, en contextos humanitarios, de ayuda, etc.), y en procesos de capacitación en las áreas comerciales y de ventas en las empresas (Peng, Lee y Heeter, 2010).

Tabla 1. Síntesis de autores y conceptualización del dominio de la empatía estudiada desde el marketing y las ventas

\begin{tabular}{|c|c|c|}
\hline Autores & Descripción de empatía & Conceptualización \\
\hline Lamont y Ludnstrom (1977) & Habilidad, comprensión cognitiva & $\begin{array}{l}\text { Habilidad para sentir la perspectiva de la } \\
\text { otra persona }\end{array}$ \\
\hline Dawson, Soper y Pettijohn (1992) & Proceso interpersonal & $\begin{array}{l}\text { Respuesta emocional congruente para per- } \\
\text { cibir el bienestar de otros }\end{array}$ \\
\hline Comer y Drollinger (1999) & Habilidad, comprensión cognitiva y emotiva & $\begin{array}{l}\text { Proceso de entendimiento de las necesida- } \\
\text { des de los clientes }\end{array}$ \\
\hline Pilling y Eroglu (1999) & Habilidad, comprensión cognitiva y emotiva & $\begin{array}{l}\text { Habilidad para sentir lo que otros sienten y } \\
\text { actuar conforme a la situación }\end{array}$ \\
\hline Aggarwal et al. (2005) & Comportamiento individual & $\begin{array}{l}\text { Las fuerzas de ventas hacen una demostra- } \\
\text { ción de interés y perspectiva por el bienes- } \\
\text { tar y la comprensión del cliente }\end{array}$ \\
\hline Giacobbe et al. (2006) & Habilidad, comprensión cognitiva y afectiva del individuo & $\begin{array}{l}\text { Habilidad para entender y comprender la } \\
\text { perspectiva del cliente }\end{array}$ \\
\hline Aheame, Jelinek y Jones (2007) & Habilidad, comprensión cognitiva y afectiva del individuo & $\begin{array}{l}\text { Habilidad para experimentar y entender a } \\
\text { la otra persona, su estado psicológico y } \\
\text { afectivo }\end{array}$ \\
\hline Fisher, Vandenbosch y Antia (2008) & Experiencia, respuesta emocional & $\begin{array}{l}\text { Habilidad para adoptar la perspectiva de } \\
\text { otros a través de la aprehensión intelectual } \\
\text { y generar sentimientos genuinos }\end{array}$ \\
\hline Argo, Zhu y Dahi (2008) & Habilidad, comprensión cognitiva y afectiva del individuo & $\begin{array}{l}\text { Los vendedores entienden a sus clientes, } \\
\text { proceso de sentimiento empático }\end{array}$ \\
\hline Homburg, Wieseke y Bornemann (2009) & Habilidad, fenómeno cognitivo & $\begin{array}{l}\text { Habilidad de los vendedores para sentir lo } \\
\text { que siente otra persona, lo que conlleva ha- } \\
\text { bilidad para vender el producto }\end{array}$ \\
\hline
\end{tabular}

Fuente: Elaboración propia. 
En el área de educación y pedagogía, las investigaciones que han implementado la adopción de perspectiva han analizado el impacto que tienen los serius games (juegos serios), que hoy están surgiendo como un nuevo medio y alternativa para propiciar cambios del orden social, en particular, el papel que cumple la adopción de perspectiva en la voluntad de ayuda. Los juegos serios son los juegos digitales, diseñados con un propósito más allá del entretenimiento, que incluye, pero no limitado, los juegos para el aprendizaje, los juegos para la salud y los juegos de promoción y cambio social (Peng, Lee y Heeter, 2010). Los resultados muestran que la adopción de perspectiva sí incide en un cambio positivo de comportamiento de la voluntad de ayuda, colaboración y comportamiento prosocial (Cho, 2009; Gee, 2009; Hota y Derbaix, 2014; Newman, 2012; Peng, Lee y Heeter, 2010; Schmitz et al., 2015).

Las investigaciones desde la psicología clínica y el desarrollo infantil (Vaish, Carpenter y Tomasello, 2009) han mostrado que los niños podrían adoptar la perspectiva con una persona a la que le haya sucedido algo negativo durante un hecho dañoso, resultado que se correlaciona positivamente con su comportamiento prosocial posterior. Los niños muy pequeños pueden simpatizar con un maltratador a pesar del daño ocasionado.

Por su parte, Farrant, Devine, Maybery y Fletcher (2012) estudian y analizan las relaciones entre la adopción de perspectiva (emocional y cognitiva), la crianza que anima al niño a tomar la perspectiva de los demás, la empatía cognitiva del niño y el comportamiento prosocial del niño. Los resultados apoyan el efecto facilitador de la crianza que anima al niño a adoptar la perspectiva de los demás. Por tanto, el papel desempeñado por los padres en el desarrollo de la conducta prosocial se extiende más allá de la crianza. Blatt, LeLacheur, Galinsky, Simmens y Greenberg (2010), en una investigación relacionada con encuentros con pacientes clínicos, concluyen que la adopción de perspectiva aumenta la satisfacción de los pacientes e impacta positivamente los encuentros interraciales, es decir, entre personas de diferentes razas.
La adopción de perspectiva, o comprensión de la vivencia del otro o de su estado afectivo o psicológico, es parte integral para alcanzar interacciones sociales positivas y sanas en las relaciones interpersonales. De hecho, la adopción de perspectiva se considera que es esencial para la aparición de comportamientos prosociales (Hoffman, 1982; De Waal, 2008; Knafo, Zahn-Waxler, Van Hulle, Robinson y Rhee, 2008). Esta afirmación teórica se ve reforzada por trabajos empíricos que demuestran que los niveles más altos de la empatía en los niños y los adultos se asocian con una mayor probabilidad de ofrecer ayuda a un extraño, la donación de dinero o la caridad (Dovidio, Allen y Schroeder, 1990; Miller, 1979), y también con una mayor disposición a encontrar y ayudar a las personas necesitadas, por ejemplo, al ser voluntario en un refugio (Davis, 2005).

\section{Consideraciones finales}

La RSC se constituye en un tema de investigación de gran interés para los académicos como para los empresarios. Particularmente, se destaca el análisis de los procesos de comunicación de la RSC, el comportamiento del consumidor y la maximización de los resultados empresariales (Du, Bhattacharya y Sen, 2010).

Las actividades de RSC son requisitos fundamentales para acceder a beneficios estratégicos de RSC y es imperativo que los administradores en las empresas tengan una comprensión más profunda de los temas claves relacionados con la comunicación de la RSC. Estos temas incluyen preguntas en torno a qué y cómo comunicar (es decir, el diseño y contenido del mensaje), dónde comunicarse (es decir, canal o medio), así como una comprensión de la condición de la empresa y, además, especificar la eficacia de la comunicación de la RSC.

Cada medio y cada formato de mensaje de comunicación tiene su propio sistema y dinámica que lo hace único, que puede incidir en el impacto y en las habilidades cognitivas que se activan para extraer significados de los mensajes (Salomon, 1979, 1990). Diferentes medios heredan ciertos factores 
para la presentación de una visión del mundo y facilitan la capacidad cognitiva para asumir roles y adoptar perspectivas.

También es importante dentro de las iniciativas de las empresas el análisis y la respuesta de los consumidores ante las estrategias de RSC y profundizar en el estudio de los efectos en el comportamiento del consumidor (Andreu, Casado-Díaz y Mattila, 2015; Du, Bhattacharya y Sen, 2007; Ellen, Webb y Mohr, 2006; Plewa et al., 2015; Sen y Bhattacharya, 2001).

La RSC en la empresa ha sido ampliamente documentada por una gran cantidad de estudios multidisciplinarios, los que han demostrado que mediante iniciativas sociales esta puede generar actitudes y comportamientos favorables de las partes interesadas (por ejemplo, compra, búsqueda de empleo, inversión en la empresa). Además, las estrategias de RSC han demostrado a largo plazo la construcción de imagen/marca corporativa, lo que ha fortalecido las relaciones con los grupos de interés y propiciado comportamientos de promoción de la empresa (por ejemplo, el boca-oído de los consumidores, que incrementa el comportamiento y el compromiso ciudadano).

Las investigaciones recientes han analizado los aspectos de la comunicación de la RSC, del contenido de los mensajes y de los canales de comunicación, así como los factores que influyen en la eficacia de la comunicación de la RSC y su incidencia en el comportamiento del consumidor. La discusión de los aspectos claves de la comunicación de la RSC también abre varias vías para la investigación (Ruiz de Maya et al., 2015).

En este sentido, las actividades de comunicación de la RSC en la empresa son un campo prolífico de la investigación por parte de académicos y profesionales en la búsqueda y compresión profunda de cómo comunicar la RSC más eficazmente a las partes interesadas.

Por ello, una vía importante para la investigación debe explorar los mecanismos mediadores que dan cuenta de la eficacia de la comunicación de la RSC. Las investigaciones relacionadas con la adopción de perspectiva han estado dirigidas a temas en la afectación en procesos de desarrollo cognitivo en la educación y su incidencia en el progreso de aprendizaje en niños y jóvenes, así como en procesos de formación en la medicina y su incidencia en la fisiología humana. Ha sido aplicada a procesos de formación en las fuerzas de ventas y en los diseños en la argumentación y negociación. Otros estudios se enfocan en cómo adoptan la perspectiva las personas involucradas en situaciones de apoyo, emergencia y ayuda médica. También las investigaciones han analizado la incidencia de la adopción de perspectiva en "juegos serios" y cómo a partir de estos las personas toman la perspectiva de otra persona en busca de la promoción en el aprendizaje, cambios de conducta y promoción social (Cho, 2009; Gee, 2009; Hota y Derbaix, 2014; Peng, Lee y Heeter, 2010; Schmitz et al., 2015). La investigación muestra una variedad de elementos cognitivos y afectivos que subyacen a la aceptación de los consumidores y, por tanto, a la efectividad de los mensajes (Batra y Ray, 1986). Por ejemplo, las formulaciones cognitivas, tales como argumentos de apoyo y refuerzos afectivos, contribuyen en la eficacia del mensaje. Según la búsqueda en la literatura académica, la adopción de perspectiva no ha sido tenida en cuenta como alternativa en la formulación de propuestas de comunicación, específicamente en la comunicación de la RSC. La investigación puede explorar opciones de origen cognitivo (por ejemplo, adopción de perspectiva) y afectivo (por ejemplo, orgullo, empatía) en busca de profundizar en la comprensión de los mecanismos psicológicos que soportan la eficacia de la comunicación de la RSC, y que, por tanto, tiene implicaciones estratégicas para la comunicación de la RSC (Du, Bhattacharya y Sen, 2010).

\section{Referencias}

Ackerman, R. W. (1973). How companies respond to social demands. Harvard Business Review, 51(4), 88-98.

Andreu, L., Casado-Díaz, A. B. y Mattila, A. S. (2015). Effects of message appeal and service type in CSR communication strategies. Journal of Business Research, 68(7), 1488-1495.

Andreu, L., Mattila, A. S. y Aldás, J. (2011). Effects of message appeal when communicating CSR initiatives. En S. Okazaki 
(ed.), Advances in advertising research (vol. 2, pp. 261275). Gabler.

Barrett-Lennard, G. T. (1981). The empathy cycle: Refinement of a nuclear concept. Journal of Counseling Psychology, 28(2), 91-100.

Batra, R. y Ray, M. L. (1986). Affective responses mediating acceptance of advertising. Journal of Consumer Research, 13(2), 234-249.

Batson, C. D. (1987). Prosocial motivation: ¿Is it ever altruistic? En L. Berkowitz (ed.), Advances in experimental social psychology (pp. 65-123). Nueva York: Academic Press.

Batson, C. D., Bolen, M. H., Cross, J. A. y Neuringer-Benefiel, H. E. (1986). Where is the altruism in the altruistic personality? Journal of Personality and Social Psychology, 50(1), 212-220.

Bhattacharya, C. B. y Sen, S. (2003). Consumer-company identification: A framework for understanding consumers' relationships with companies. Journal of Marketing, 67(2), 76-88.

Bhattacharya, C. B. y Sen, S. (2004). Doing better at doing good: When, why, and how consumers respond to corporate social initiatives. California Management Review, 47(1), 9-24.

Bhattacharya, C. B., Rao, H. y Glynn, M. A. (1995). Understanding the bond of identification: An investigation of its correlates among art museum members. Journal of Marketing, 59(4), 46-57.

Bigné-Alcañiz, E., Chumpitaz, R., Andreu, L. y Swaen, V. (2005). Percepción de la responsabilidad social corporativa: un análisis cross-cultural. Universia Business Review, 1(5), 14-27.

Bigné-Alcañiz, E. y Currás-Pérez, R. (2008). ¿Influye la imagen de responsabilidad social en la intención de compra? El papel de la identificación del consumidor con la empresa. Universia Business Review, 19, 10-23.

Blatt, B., LeLacheur, S. F., Galinsky, A. D., Simmens, S. J. y Greenberg, L. (2010). Does perspective-taking increase patient satisfaction in medical encounters? Academic Medicine, 85(9), 1445-1452.

Brown, T. J. y Dacin, P. A. (1997). The company and the product: Corporate associations and consumer product responses. The Journal of Marketing, 61(1), 68-84.

Carroll, A. B. (1979). A three-dimensional conceptual model of corporate performance. Academy of Management Review, 4(4), 497-505.

Carroll, A. B. (1999). Corporate social responsibility: Evolution of a definitional construct. Business \& Society, 38(3), 268-295.

Cho, J. (2009). Disentangling media effects from debate effects: The presentation mode of televised debates and viewer decision making. Journalism \& Mass Communication Quarterly, 86(2), 383-400.

Cohen, J. (2001). Defining identification: A theoretical look at the identification of audiences with media characters. Mass Communication \& Society, 4(3), 245-264.

Cohen, T. R. (2010). Moral emotions and unethical bargaining: The differential effects of empathy and perspective-taking in deterring deceitful negotiation. Journal of Business Ethics, 94(4), 569-579.
Commission of the European Communities (2001). Promoting a european framework for corporate social responsibilities. Bruselas: Commission of the European Communities.

Coplan, A. (2011). Will the real empathy please stand up? A case for a narrow conceptualization. The Southern Journal of Philosophy, 49, 40-65.

Coutu, W. (1951). Role-playing vs. role-taking: An appeal for clarification. American Sociological Review, 16(2), 180-187.

Cox, C. L., Uddin, L. Q., Di Martino, A., Castellanos, F. X., Milham, M. P. y Kelly, C. (2011). The balance between feeling and knowing: Affective and cognitive empathy are reflected in the brain's intrinsic functional dynamics. Social Cognitive and Affective Neuroscience, 7(6), 727-737.

Creyer, E. H. (1997). The influence of firm behavior on purchase intention: Do consumers really care about business ethics? Journal of consumer Marketing, 14(6), 421-432.

Cuff, B. M., Brown, S. J., Taylor, L. y Howat, D. J. (2016). Empathy: A review of the concept. Emotion Review, 8(2), 144-153.

Davis, M. H. (1980). A multidimensional approach to individual differences in empathy. Catalog of Selected Documents in Psychology, 10(85), 1-17.

Davis, M. H. (1996). A social psychological approach. Boulder: Westview Press.

Davis, M. H. (2005). Becoming (and remaining) a community volunteer: Does personality matter. En A. M. Omoto (ed.), Processes of community change and social action (pp. 6782). Mahwah, NJ: Lawrence Erlbaum Associates Publishers.

Dawkins, J. (2005). Corporate responsibility: The communication challenge. Journal of Communication Management, 9(2), 108-119.

Decety, J. (2010). To what extent is the experience of empathy mediated by shared neural circuits? Emotion Review, 2(3), 204-207.

Decety, J. (2011). Dissecting the neural mechanisms mediating empathy. Emotion Review, 3(1), 92-108.

Decety, J. y Chaminade, T. (2003). When the self represents the other: A new cognitive neuroscience view on psychological identification. Consciousness and Cognition, 12(4), 577-596.

Decety, J. y Cowell, J. M. (2014a). Friends or foes: Is empathy necessary for moral behavior? Perspectives on Psychological Science, 9(5), 525-537.

Decety, J. y Cowell, J. M. (2014b). The complex relation between morality and empathy. Trends in Cognitive Sciences, 18(7), 337-339.

De Waal, F. B. (2008). Putting the altruism back into altruism: The evolution of empathy. Annual Review of Psychology, 59, 279-300.

Delpechitre, D. (2013). Review and assessment of past empathy scales to measure salesperson's empathy. Journal of Management and Marketing Research, 13.

Devoldre, I., Davis, M. H., Verhofstadt, L. L. y Buysse, A. (2010). Empathy and social support provision in couples: Social support and the need to study the underlying processes. The Journal of psychology, 144(3), 259-284. 
Dovidio, J. F., Allen, J. L. y Schroeder, D. A. (1990). Specificity of empathy-induced helping: Evidence for altruistic motivation. Journal of Personality and Social Psychology, 59(2), 249-206.

Dymond, R. F. (1949). A scale for the measurement of empathic ability. Journal of Consulting Psychology, 13(2), 127-133.

Du, S., Bhattacharya, C. B. y Sen, S. (2007). Reaping relational rewards from corporate social responsibility: The role of competitive positioning. International Journal of Research in Marketing, 24(3), 224-241.

Du, S., Bhattacharya, C. B. y Sen, S. (2010). Maximizing business returns to corporate social responsibility (CSR): The role of CSR communication. International Journal of Management Reviews, 12(1), 8-19.

Eisenberg, N. y Miller, P. A. (1987). The relation of empathy to prosocial and related behaviors. Psychological Bulletin, 101(1), 91-119.

Eisenberg, N. y Morris, A. S. (2001). The origins and social significance of empathy-related responding. A review of empathy and moral development: Implications for caring and justice by ML Hoffman. Social Justice Research, 14(1), 95-120.

Eisenberg, N., Fabes, R. A. y Spinrad, T. L. (2006). Prosocial development. En W. Damon, R. M. Lerner y N. Eisenberg (eds.), Handbook of child psychology, social, emotional, and personality development (6. ${ }^{\mathrm{a}}$ ed., vol. 3, pp. 646-718). Hoboken: John Wiley y Sons.

Eisenberg, N., Zhou, Q., Spinrad, T. L., Valiente, C., Fabes, R. A. y Liew, J. (2005). Relations among positive parenting, children's effortful control, and externalizing problems: A three-wave longitudinal study. Child Development, 76(5), 1055-1071.

Eklund, J., Andersson-Stråberg, T. y Hansen, E. M. (2009). "I've also experienced loss and fear": Effects of prior similar experience on empathy. Personality and Social Sciences, 50(1), 65-69.

Ellen, P. S., Webb, D. J. y Mohr, L. A. (2006). Building corporate associations: Consumer attributions for corporate socially responsible programs. Journal of the Academy of Marketing Science, 34(2), 147-157.

Epley, N., Caruso, E. M. y Bazerman, M. H. (2006). When perspective-taking increases taking: Reactive egoism in social interaction. Journal of Personality and Social Psychology, 91(5), 872-889.

Fan, Y., Duncan, N. W., de Greck, M. y Northoff, G. (2011). Is there a core neural network in empathy? An fMRI based quantitative meta-analysis. Neuroscience \& Biobehavioral Reviews, 35(3), 903-911.

Farrant, B. M., Devine, T. A., Maybery, M. T. y Fletcher, J. (2012). Empathy, perspective-taking and prosocial behaviour: The importance of parenting practices. Infant and Child Development, 21(2), 175-188.

Fazio, R. H. (1990). Multiple processes by which attitudes guide behavior: The mode model as an integrative framework. Advances in Experimental Social Psychology, 23, 75-109.

Freeman, R. E. (1984). Strategic management: A stakeholder perspective. Boston: Pitman.

Galinsky, A. D. y Moskowitz, G. B. (2000). Perspective-taking: Decreasing stereotype expression, stereotype accessibility, and in-group favoritism. Journal of Personality and Social Psychology, 78(4), 708-724.

Galinsky, A. D., Ku, G. y Wang, C. S. (2005). Perspective-taking and self-other overlap: Fostering social bonds and facilitating social coordination. Group Processes \& Intergroup Relations, 8(2), 109-124.

Galinsky, A. D., Magee, J. C., Gruenfeld, D. H., Whitson, J. A. y Liljenquist, K. A. (2008). Power reduces the press of the situation: Implications for creativity, conformity, and dissonance. Journal of Personality and Social Psychology, 95(6), 1450-1466.

Galinsky, A. D., Magee, J. C., Inesi, M. E. y Gruenfeld, D. H. (2006). Power and perspectives not taken. Psychological Science, 17(12), 1068-1074.

Galinsky, A. D., Wang, C. S. y Ku, G. (2008). Perspective-takers behave more stereotypically. Journal of Personality and Social Psychology, 95(2), 404-419.

Gee, J. P. (2009). Deep learning properties of good digital games: How far can they go? En U. Ritterfeld, M. Cody y P. Vorderer (eds.), Serious games: Mechanisms and effects (pp. 89-104). Nueva York: Routledge.

Gilin, D., Maddux, W. W., Carpenter, J. y Galinsky, A. D. (2013). When to use your head and when to use your heart: The differential value of perspective-taking versus empathy in competitive interactions. Personality and Social Psychology Bulletin, 39(1), 3-16.

Harjoto, M. A. y Jo, H. (2011). Corporate governance and CSR nexus. Journal of Business Ethics, 100(1), 45-67.

Hoeffler, S. y Keller, K. L. (2002). Building brand equity through corporate societal marketing. Journal of Public Policy \& Marketing, 21(1), 78-89.

Hoffman, M. L. (1982). Affect and moral development. New Directions for Child and Adolescent Development, 1982(16), 83-103.

Hota, M, y Derbaix. M. (2014) A real child in a virtual world: Children's motives for participating in virtual gaming communities. IESEG working series, MKT-01.

International Organization for Standardization (2010). ISO 26000:2010-Guidance on social responsibility. Recuperado de http://www.iso.org/iso/catalogue_detail?csnumber=42546

Johnston, M. W. y Marshall, G. W. (1998). Administración de ventas (2. ${ }^{a}$ ed.). México: McGraw-Hill.

Kalliopuska, M. (1986). Empathy and the experiencing of loneliness. Psychological Reports, 59(3), 1052-1054.

Kelley, H. H. y Thibaut, J. W. (1978). Interpersonal relations: A theory of interdependence. Nueva York: Wiley.

Klein, J. y Dawar, N. (2004). Corporate social responsibility and consumers' attributions and brand evaluations in a productharm crisis. International Journal of Research in Marketing, 21(3), 203-217.

Knafo, A., Zahn-Waxler, C., Van Hulle, C., Robinson, J. L. y Rhee, S. H. (2008). The developmental origins of a disposition toward empathy: Genetic and environmental contributions. Emotion, 8(6), 737-752. 
Lantos, G. P. (2002). The ethicality of altruistic corporate social responsibility. Journal of Consumer Marketing, 19(3), 205-232.

Lishner, D. A., Batson, C. D. y Huss, E. (2011). Tenderness and sympathy: Distinct empathic emotions elicited by different forms of need. Personality and Social Psychology Bulletin, 37(5), 614-625.

Lindgreen, A., Swaen, V. y Johnston, W. J. (2009). Corporate social responsibility: An empirical investigation of US organizations. Journal of Business Ethics, 85(2), 303-323.

Litchfield, R. C. y Gentry, R. J. (2010). Perspective-taking as an organizational capability. Strategic Organization, 8(3), 187-205.

Marín, L. y Ruiz, S. (2008). La evaluación de la empresa por el consumidor según sus acciones de RSC. Cuadernos de Economía y Dirección de la Empresa, 11(35), 91-112.

Marín, L., Cuestas, P. J. y Román, S. (2016). Determinants of consumer attributions of corporate social responsibility. Journal of Business Ethics, 138(2), 247-260.

Marín, L., Rubio, A. y de Maya, S. R. (2012). Competitiveness as a strategic outcome of corporate social responsibility. Corporate Social Responsibility and Environmental Management, 19(6), 364-376.

McBane, D. A. (1995). Empathy and the salesperson: A multidimensional perspective. Psychology \& Marketing, 12(4), 349-370.

Moura-Leite, R. C. y Padgett, R. C. (2011). Historical background of corporate social responsibility. Social Responsibility Journal, 7(4), 528-539.

Newman, G. L. (2012). "Now imagine you're one of them": Using serious games to induce identification with out-groups. Masters Theses \& Specialist Projects, 1224. Recuperado de https://digitalcommons.wku.edu/cgi/viewcontent. cgi?referer=https://scholar.google.es/\&httpsredir=1\&articl $e=2227 \&$ context $=$ theses

Parker, S. K. y Axtell, C. M. (2001). Seeing another viewpoint: Antecedents and outcomes of employee perspective-taking. Academy of Management Journal, 44(6), 1085-1100.

Peng, W., Lee, M. y Heeter, C. (2010). The effects of a serious game on role-taking and willingness to help. Journal of Communication, 60(4), 723-742.

Perner, J., Leekam, S. R. y Wimmer, H. (1987). Three-year-olds' difficulty with false belief: The case for a conceptual deficit. British Journal of Developmental Psychology, 5(2), 125-137.

Piliavin, I. M., Rodin, J. y Piliavin, J. A. (1969). Good samaritanism: An underground phenomenon? Journal of Personality and Social Psychology, 13(4), 289-299.

Piliavin, J. A., Dovidio, J. F., Gaertner, S. L. y Clark, R. D. III. (1981). Emergency Intervention. Nueva York: Academic.

Pomering, A. y Dolnicar, S. (2009). Assessing the prerequisite of successful CSR implementation: Are consumers aware of CSR initiatives? Journal of Business Ethics, 85(2), 285-301.

Porter, M. y Kramer, M. (2006). Strategy and society: The link between competitive advantage and corporate social responsibility. Harvard Business Review, 84(12), 78-92.

Pryor, S., Malshe, A. y Paradise, K. (2013). Salesperson listening in the extended sales relationship: An exploration of cognitive, affective, and temporal dimensions. Journal of Personal Selling \& Sales Management, 33(2), 185-196.

Rogers, C. R. (1975). Empathic: An unappreciated way of being. The Counseling Psychologist, 5(2), 2-10.

Sen, S. y Bhattacharya, C. B. (2001). Does doing good always lead to doing better? Consumer reactions to corporate social responsibility. Journal of Marketing Research, 38(2), 225-243.

Sen, S., Bhattacharya, C. B. y Korschun, D. (2006). The role of corporate social responsibility in strengthening multiple stakeholder relationships: A field experiment. Journal of the Academy of Marketing Science, 34(2), 158-166.

Sheehy, B. (2015). Defining CSR: Problems and solutions. Journal of Business Ethics, 131(3), 625-648.

Smith, A. (1759). The theory of moral sentiments. Londres: A. Miller.

Strack, F. y Deutsch, R. (2004). Reflective and impulsive determinants of social behavior. Personality and Social Psychology Review, 8(3), 220-247.

Vaish, A., Carpenter, M. y Tomasello, M. (2009). Sympathy through affective perspective-taking and its relation to prosocial behavior in toddlers. Developmental Psychology, 45(2), 534-543.

Webb, D. J. y Mohr, L. A. (1998). A typology of consumer responses to cause-related marketing: From skeptics to socially concerned. Journal of Public Policy y Marketing, 17(2), 226-238.

Wieseke, D. J., Geigenmüller, A. y Kraus, F. (2012). On the role of empathy in customer-employee interactions. Journal of Service Research, 15(3), 316-331.

Wispé, L. (1986). The distinction between sympathy and empathy: To call forth a concept, a word is needed. Journal of Personality and Social Psychology, 50(2), 314-321.

Wood, D. J. (1991). Corporate social performance revisited. Academy of Management Review, 16(4), 691-718.

Wood, D. J. (2010). Measuring corporate social performance: A review. International Journal of Management Reviews, 12(1), 50-84.

Wondra, J. D. y Ellsworth, P. C. (2015). An appraisal theory of empathy and other vicarious emotional experiences. Psychological Review, 122(3), 411-428.

Watts, P. y Holme, R. (1999). Corporate social responsibility: Meeting changing expectations. Génova: World Business Council for Sustainable Development.

Wouters, P., van Nimwegen, C., van Oostendorp, H. y van der Spek, E. D. (2013). A meta-analysis of the cognitive and motivational effects of serious games. Journal of Educational Psychology, 105(2), 249-265.

Yang, X. y Rivers, C. (2009). Antecedents of CSR practices in MNCs' subsidiaries: A stakeholder and institutional perspective. Journal of Business Ethics, 86(2), 155-169.

Yoon, Y., Gürhan-Canli, Z. y Schwarz, N. (2006). The effect of corporate social responsibility (CSR) activities on companies with bad reputations. Journal of Consumer Psychology, 16(4), 377-390.

Zaki, J. (2014). Empathy: A motivated account. Psychological Bulletin, 140(6), 1608-1647. 